\title{
Virulence Factors and Pathogencity Islands in Staphylococcus Species: A Review
}

\author{
Desiye Tesfaye Tegegne* \\ Animal Biotechnology Research Program, National Agricultural Biotechnology Research Center, Ethiopian \\ Institute Of Agricultural Research, P.O. Box 249, Holeta, Ethiopia
}

*Corresponding Author: Desiye Tesfaye Tegegne, Animal Biotechnology Research Program, National Agricultural Biotechnology Research Center, Ethiopian Institute Of Agricultural Research, P.O. Box 249, Holeta, Ethiopia,

\begin{abstract}
Staphylococcus aureus (S.aureus) is a common pathogen associated with serious community and hospital acquired diseases and has long been considered as a major problem of public health. This potent Gram-positive bacterium is able to bypass all barriers of the host defense system as it possesses a wide spectrum of virulence factors. S. aureus is also one of the prominent pathogens in biofilm-related infections of indwelling medical devices, which are responsible for billions in healthcare cost each year in developing countries. S. aureus expresses a large number of virulence factors that are implicated in their pathogenesis. Methicillin-resistant S. aureus infections have reached epidemic levels in many parts of the world. This review describes the virulence factors and pathogenic islands in major pathogenic staphylococcus especially S.aureus
\end{abstract}

Keywords: Staphylococcus aureus, pathogenic islands, virulence factor

\section{INTRODUCTION}

Staphylococci are gram positive bacteria, 0.5-1.5 $\mu \mathrm{m}$ in diameter and appear as individual coccus, in pairs, tetrads or in grape like clusters. The genus Staphylococcus has 41 species many of which colonize human and animal body. S. aureus species which is well characterized and have been studied in detail. These organisms are non-motile, non-spore forming, facultative anaerobes that grow by aerobic respiration or by fermentation. Their growth is optimum at $37^{\circ} \mathrm{C}$ but yellow pigment formation can be seen at room temperature $\left(20-25^{\circ} \mathrm{C}\right)$. Colonies of $S$. aureus are round, smooth with entire margin, opaque, raised and glistening on solid media. Most species of this genus require complex nutrition, but in general, they require nitrogen as organic source, which is supplied by amino acids[1]. Staphylococci have the ability to tolerate high salt concentration [2]. Members of the genus Staphylococcus are catalase positive and oxidase negative. The catalase test differentiates Staphylococci from Streptococci. These genera also differ in the composition of their cell walls. Pathogenic Staphylococci such as $S$. aureus can generally be identified by their ability to produce coagulase enzyme. The coagulase negative Staphylococcus(CNS) are commensals or saprophytic but some of them can cause opportunistic infections [3]

Staphylococcus aureus is a major human and animal opportunistic pathogen responsible for nosocomial and community-associated infections with high morbidity and mortality. This potent Gram-positive bacterium is able to bypass all barriers of the host defense system as it possesses a wide spectrum of virulence factors. About one in three healthy individuals is asymptomatically colonized intranasally with S. aureus without any associated disease. However, S. aureus carriers are at high risk of infection, and they are presumed to be an important source of the S. aureus strains that spread among related individuals and the community at large. The objectives of this review are to 
highlight the virulence factors, secretion system and pathogencity islands and other related issue about major pathogenic staphylococcus species especially staphylococcus aureus. 


\section{CHARACTERISTICS OF THE ORGANISM}

Recently, the taxonomy of the phylum Firmicutes, to which the staphylococci belong, has been completely revised. The genus Staphylococcus belongs to the class Bacilli, order Bacillales, family Staphylococcaceae, together with the general Macrococcus Jeotgalicoccus and Salinicoccus. The minimal standards for assigning an organism to the genus Staphylococcus include genotypic as well as phenotypic criteria[4] .

\subsection{Genotypic Criteria}

The DNA of staphylococci has a guanine plus cytosine $(\mathrm{G}+\mathrm{C})$ content of $30-39 \mathrm{~mol} \%$. Other genotypic criteria to assign an unknown species to the genus Staphylococcus are based on phylogenetic trees constructed by comparison of $16 \mathrm{~S}$ rRNA or 23S rRNA sequences.

The staphylococci can be divided by 16S rRNA gene sequence analysis[4] and whole - genome DNA - DNA reassociation studies into several phylogenetic species groups. Species within these groups typically share important characteristics, are usually difficult to differentiate from each other, and may occupy similar ecological niches or show similar pathogencity potentials.

To some degree, the staphylococci have evolved along with their host species. Different animal species have different staphylococcal micro flora. This can be seen at the species level and at the strain level. At the infraspecies level, genotypic studies have characterized staphylococcal species in epidemiological (fingerprinting) studies at different levels up to the strain level. From the veterinary and zoonotic perspectives, typing methods distinguishing between host- associated groups of strains (ecovars) can be useful. However, the host association is not absolute since it has been shown that certain clonal strains can colonize different animal species, as is, for instance, the case for the so called animal - associated methicillin - resistant Staphylococcus aureus(MRSA).

\subsection{Phenotypic Criteria}

The catalase reaction is positive for all Staphylococcus species, except for S. aureus subsp. anaerobius and Staphlococcus saccharolyticus. Staphylococci are facultative anaerobic bacteria, except for $S$. aureus subsp. anaerobius and $S$. saccharolyticus, which are strictly anaerobic. The ultrastructure and chemical composition of the cell wall of staphylococci are those as typical for gram - positive bacteria. The wall is made up of peptidoglycans, teichoic acid, and proteins [4].

On the basis of the coagulase test, the genus coagulase - positive species $S$. aureus and coagulase negative staphylococci (CNS). In humans, staphylococci are among the most common causes of bacterial infections, and $S$. aureus is the most important pathogenic Staphylococcus species. In veterinary medicine, it used to be stated that three staphylococcal species were of major pathogenic importance, namely, S. aureus, Staphylococcus hyicus, and Staphylococcus intermedius. This statement stays correct for $S$. aureus and $S$. hyicus, but following the description of a new species, Staphylococcus pseudintermedius. It appears that strains formerly identified as $S$. intermedius should be reclassified. All canine and some equine staphylococci previously identified as $S$. intermedius are now considered to be $S$. pseudintermedius. Other previous $S$. intermedius strains (from, e.g., domestic pigeons and horses) appear to belong to the species Staphylococcus delphini. Real S. intermedius strains seem to be confined to feral pigeons. Phenotypic tests offer some help in the differentiation of S. intermedius, S. pseudintermedius, and $S$. delphini. Unlike the other two species, S. intermedius acidifies $\beta$ - gentiobiose and D - mannitol but fails to react in arginine dihydrolase tests. Phenotypic distinction between S. delphini and S. pseudintermedius is difficult. Non - canine isolates are probably better reported as strains belonging to the $S$. intermedius species group[5].

Because of this complex situation, it is more convenient to use the terms "major" and "minor" pathogenic staphylococci than coagulase - positive and coagulase - negative staphylococci. This terminology has been adopted for many years in mastitis bacteriology. The major pathogenic species are $S$. aureus, S. hyicus, while all others are to be considered minor pathogens. In veterinary medicine, the latter are mainly of importance in subclinical mastitis, while the former play a role in a multitude of well - recognizable pathological conditions in many different animal species. However, the minor pathogenic staphylococci can be involved in severe disease affecting debilitated patients in hospital conditions. To date, this has only been well documented in human hospitals, but it may become more common as complex surgery and care of aged patients become more common in companion animal medicine[5]. 


\section{Major Pathogenic Staphylococcus Species}

In humans, $S$. aureus is a well-documented opportunistic pathogen. It may cause skin infections, as well as enterotoxemia and septicemic infections, and is furthermore important as the cause of toxic shock syndrome (TSS). S. aureus infections may be community acquired but are also important as nosocomial infections. An important impediment in the control of $S$. aureus infections is antimicrobial resistance. MRSAs are a major clinical and epidemiological problem in human hospitals. MRSAs have a tendency to accumulate, in addition to their methicillin - resistance gene, additional unrelated resistance determinants in their genome. This has led to the evolution of MRSA strains resistant to almost all commonly used antimicrobial agents. From the original description of MRSA in animals [6]to the end of the 20th century, only MRSA strains showing characteristics commonly seen in human epidemic strains were isolated. Animals apparently were infected by their human attendants. More recently, a so - called animal-associated MRSA strain has emerged in different animal species. This strain, characterized with multilocus sequence typing (MLST) to belong to the clonal complex ST 398, was first isolated in pig farms but has also been revealed in poultry [7] and horses [8]and appears to be present throughout Europe. This clone is often transmitted from animals to humans and has important zoonotic significance.

Typical animal strains of $S$. aureus are important pathogens in veterinary medicine, causing disease in cattle, small ruminants, poultry, rabbits, pigs, and horses[9] and in many other animal species as well. In cattle and small ruminants, $S$. aureus is one of the major causes of mastitis. Joint infections, osteomyelitis, and septicemia due to $S$. aureus are described in poultry [10]. In rabbits, S. aureus mainly causes mastitis, pustular dermatitis, subcutaneous abscesses, and pododermatitis. In horses, the bacterium may cause dermatitis and cellulitis [11] Pigs may sporadically suffer from septicemia due to a $S$. aureus infection[9]. The ST 398 MRSA strain has also been described as a cause of exudative epidermitis in pigs[12].

Staphylococcus pseudintermedius and the other species of the SIG are the most important non aureus species. S. pseudintermedius is the predominant coagulase - positive Staphylococcus causing skin infections in dogs[13] .Species from the SIG may also cause septicemia in ducks and pigeons and dermatitis in mink and horses[14]. Exudative epidermitis (greasy pig disease) and sporadic joint infections or cystitis in pigs are mostly caused by S. hyicus[14].This species appears to be involved also in flank biting and ear - tip necrosis syndrome[15]. S. hyicus plays an important role in exudative skin infections of cattle (secondary to mange), horses, and even poultry (secondary to pox lesions). Nearly all phenotypic studies at the infraspecies level have been carried out with $S$. aureus strains. Phage typing has been extraordinarily useful in epidemiologically important characterizations of human strains and continues to be so, supplemented by molecular fingerprinting methods. Phage typing has been used with animal strains of $S$. aureus and with other staphylococcal species, but the methods are labor intensive and phage supplies are difficult to standardize. Therefore, molecular methods are probably better suited for veterinary strains. Up until now, pulsed field gel electrophoresis (PFGE) remains the standard procedure for these molecular methods. However, spa typing and MLST are also applied quite regularly for animal strains, especially in the case of MRSA.

\section{Staphylococcal Virulence Factors ANd PATHOGENESIS}

The broad range of infections caused by $\mathrm{S}$. aureus is related to a number of virulence factors that allow it to adhere to surface, invade, or avoid the immune system, and cause harmful toxic effects to the host[16]. Infections caused by S. aureus can be divided into three types: superficial lesions such as wound infection; toxinoses such as food poisoning, scalded skin syndrome, and toxic shock syndrome toxin (TSST); and systemic and life-threatening conditions such as endocarditis, osteomyelitis, pneumonia, brain abscesses, meningitis, and bacteremia[17]. The infections usually occur as a consequence of $\mathrm{S}$. aureus inoculation into an open wound. Alternatively, in the upper airway, viral infection damages mucosal linings and predisposes the host to S. aureus pneumonia, which classically presents a week after onset of influenza infection. After initial exposure of S. aureus to the host tissues (beyond the mucosal surface or skin), upregulation of S. aureus virulence genes will be triggered. Accordingly, the host phagocytes and epithelial cells in the skin or mucosal tissue respond to either bacterial products or tissue injury by activation of the immune system[17].

S. aureus can overcome the host complement and antibodies, which directly or indirectly leads to killing of S. aureus by expressing on its surface a capsule, clumping factor A, protein A, and a number 
of complement inhibitors, all of which inactivate or prevent host opsonins from bacterium destruction[18,19]. Host neutrophils present more challenge to S. aureus; however, it secretes two molecules, CHIP (chemotaxis inhibitory protein) and Eap (extracellular adherence protein), which block neutrophil recognition of chemotactic factors and neutrophil binding to cell adhesion molecule, which respectively prevents leukocyte adhesion and resist neutrophil killing. MRSA produces phenolsoluble modulin, which is a group of bacterial toxins that induce inflammation and neutrophil cytolysis. Additionally, staphylococcal enterotoxins, TSST, and Eap could all alter T cell functions by targeting the $\mathrm{T}$ cell receptor activation pathway. This tactic can prevent the development of long-term memory and that can explain the ability of S. aureus to infect the human host repeatedly throughout life[20].

Many virulence factors contribute to the pathogenesis of staphylococcal infections. The expression of these virulence factors is precisely tuned by regulatory proteins and RNAs[20]. The best known global regulator is the accessory gene regulator (agr). agr was identified as a quorum sensing system that enables cell-to-cell communication and regulation of numerous colonization and virulence factors. It controls a large set of genes, including most of those encoding cell-wall-associated and extracellular proteins[20]. The agr locus consists of two divergent operons driven by the P2 and P3 promoters. The P2 operon contains agrBDCA and codes for the RNAII transcript. P3 drives transcription of RNAIII, which is the effector molecule of the agr system and controls the switch between the expression of surface proteins and excreted toxins. AgrA and AgrC comprise a twocomponent regulatory system in which the agrC (the signal receptor) binds the extracellular autoinducing peptide (AIP), which is the signaling molecule coded by agrD and processed by agrB, and modulates the activity of agrA (the response regulator). AgrA activity then leads to greatly increased P2 and P3 transcription. Signaling via this system results in an elevated intracellular concentration of RNAIII, which acts to increase the expression of a series of toxins and virulence factors[21]

Staphylococcus aureus virulence factors are divided according to their mechanisms into: invasion and inflammation virulence factors that include colonization, synthesis of extracellular molecules that facilitate adherence and the ability to evade host defenses; secreted virulence factors such as toxins, which are responsible for damaging host tissue and promoting dissemination; and ability to form biofilms, which is important in certain infections[22]

\section{BIOFILM FORMATION}

Bacterial biofilms are a complex microenvironment consisting of a single or mixed species that are attached to one another or to surfaces and encased within extracellular polymeric substances (EPSs). The key characteristics of biofilm-forming bacteria are their resistance to host defenses and their tolerance of antimicrobials[16]. S. aureus is one of the most common pathogens in biofilm-related infections of indwelling medical devices, which are responsible for billions in healthcare costs each year in the United States[23]. This exceptional status among biofilm-associated pathogens is because of the fact that staphylococci are frequent commensal bacteria on the human skin and mucus surfaces. Thus, staphylococci are among the most likely germs to infect any medical device that penetrates those surfaces, such as when being inserted during surgery[23].

S. aureus also has an inherent ability to form biofilms on biotic and abiotic surfaces. The structures of these biofilms range from a monolayer of scattered single cells to a thick mucus multilayered structure with channels allowing liquid and gas flow and dispersion of nutrients and waste components. Moreover, the chemical composition within the biofilm sludge is highly dynamic; it promotes solute gradient formation and nutrient exchange[24].

Biofilm formation is a multistep process, starting with transient adherence to a surface. Subsequently, specific bacterial adhesins, referred to as MSCRAMMS, promote the actual attachment. Next, during the accumulation phase, bacteria stick to each other and production of EPS and/or incorporation of host-derived components, such as platelets, take place, resulting in a mature biofilm. Once biofilm is established, sessile bacteria express genes in a pattern that greatly differs from their planktonic unattached individual cells - counterparts, leading to phenotypic changes. In circumstances of nutrient deprivation, or under heavy shear forces, detachment of bacteria occurs through autonomous formation of AIPs, with release and dispersal of bacteria as a consequence[25]. Sessile bacteria are particularly resistant to antibiotic treatment. In addition to the difficulty of effectively inhibiting 
biofilm with conventional antibiotic therapy, treatment is further complicated by the increase of antibiotic resistance among Staphylococci[23].

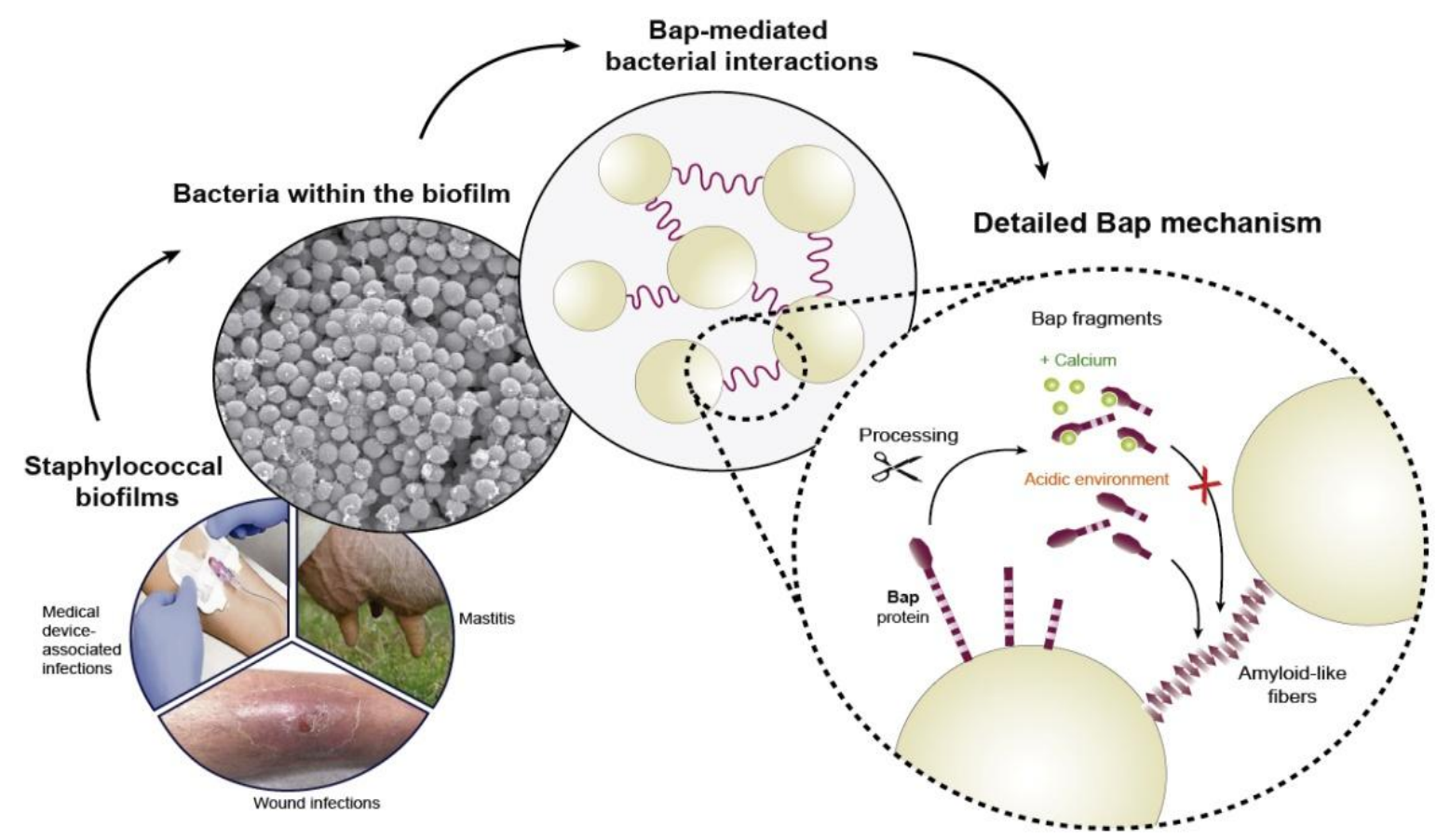

Figure1. S.aureus can cause many infections through the formation of a bio film adhered to different alive and inert surfaces

Sources:- [23]

A deep look into some of these biofilms reveals the presences of millions of bacterial glued together by an extracellular matrix composed by proteins. Particularly, the bap protein described in our work is capable, under acidic and low calcium conditions, to form amyloid-like fibers that are ultimately responsible for the formation of a biofilm in S.aureus.

\section{Pathogenicity Islands (Pais)}

Pathogenicity islands (PAIs) are distinct genetic elements of pathogens encoding various virulence factors, and are a subset of genomic islands (GIs) which mediate the horizontal transfer of genes encoding numerous virulence factors such as type III secretion system. They include type III secretion system and host invasion (LEE PAI in pathogenic Escherichia coli, Hrp PAI in Pseudomonas syringae), superantigen (SaPI1 and SaPI2 in Staphylococcus aureus), colonization factor (VPI in Vibrio cholerae), iron uptake system (SHI-2 in Shigella flexneri), and enterotoxin (espC PAI in Escherichia coli, she PAI in Shigella flexneri) [26]. Although PAI is loosely defined concept, several criteria were suggested to identify PAI: i) Encoding for virulence factors such as toxins, adhesins and invasins, ii) Presence in pathogenic strains, and absence in non-pathogenic strains of one species or a related species, iii) Different $\mathrm{G}+\mathrm{C}$ content and codon usage from the rest of the chromosome, iv) Large genomic regions (often $>30 \mathrm{~kb}$ ), v) Association with tRNA genes and/or insertion sequence (IS) elements at their boundaries, vi) Instability. PAIs are a subset of genomic islands (GIs) which have been transferred by horizontal gene transfer (HGT) event and confer virulence upon the recipient. Identification of PAIs is essential in understanding the development of disease and the evolution of bacterial pathogenesis[27].

The DNA and amino acid sequences of a genome are processed as follows. (1) Genomic regions homologous to PAI and REI loci are identified by BLAT and BLAST searches against PAIDB. (2) The existence of known virulence and resistance genes in a genomic region is checked through BLASTP searches against VFDB, CARD and BacMet databases. (3) The PAI-like region is examined for overlapping GIs through detection of HGT regions[28]. 


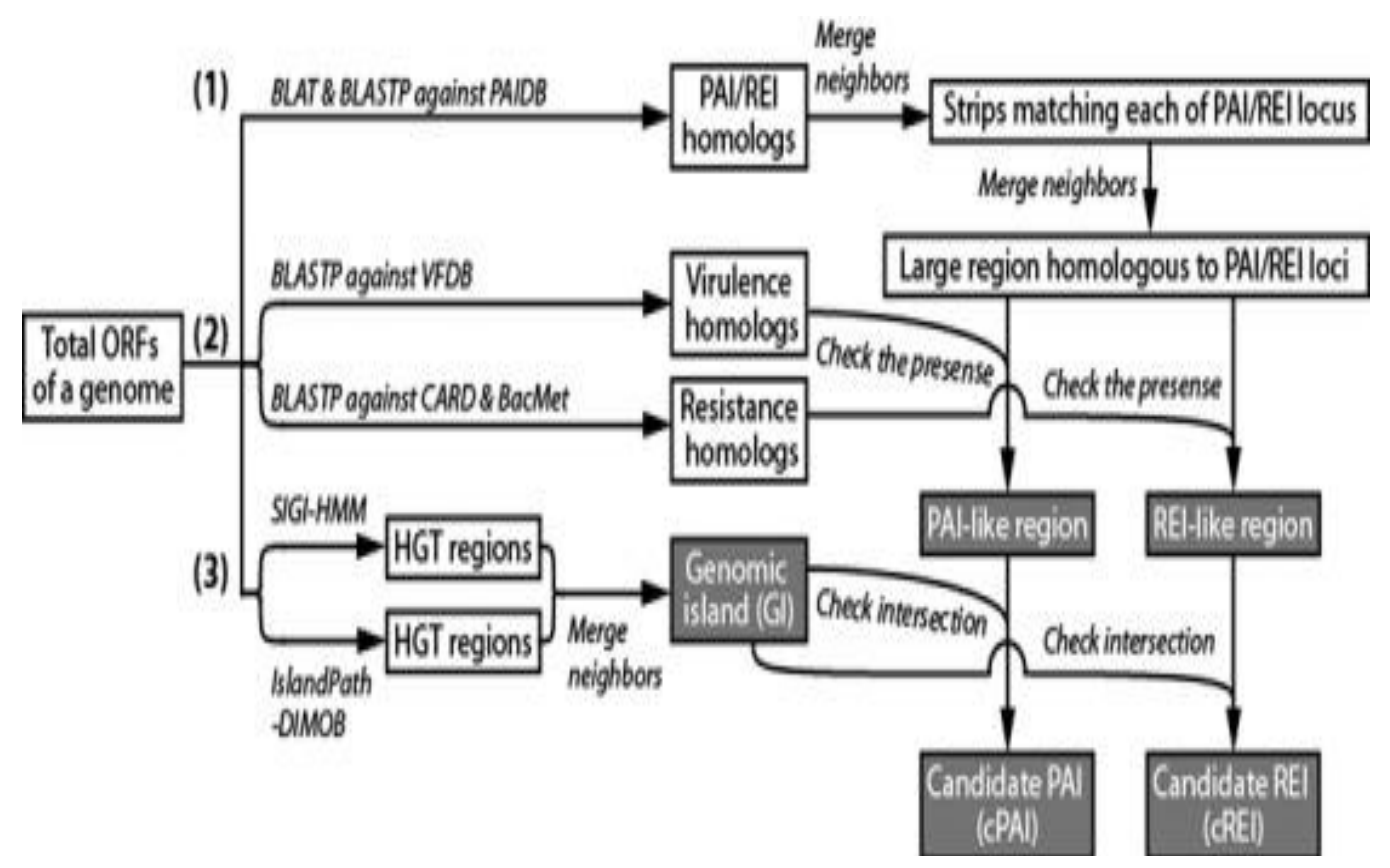

From Pathogenic Islands Database(PAIDB)

Figure2. Procedure for identifying candidate PAIs and REIs in a sequenced genome

\section{REFERENCES}

[1] Resch-Genger U, Grabolle M, Cavaliere-Jaricot S, Nitschke R, Nann T. Quantum dots versus organic dyes as fluorescent labels. Nat Methods. Nature Publishing Group; 2008;5:763.

[2] Kloos We, Wolfshohl F. Staphylococcus cohnii subspecies: Staphylococcus cohnii subsp. cohnii subsp. nov. and Staphylococcus cohnii subsp. urealyticum subsp. nov. Int J Syst Evol Microbiol. Microbiology Society; 1991;41:284-9.

[3] Rooijakkers SHM, Ruyken M, Roos A, Daha MR, Presanis JS, Sim RB, et al. Immune evasion by a staphylococcal complement inhibitor that acts on C3 convertases. Nat Immunol. Nature Publishing Group; 2005;6:920.

[4] Freney J, Kloos WE, Hajek V, Webster JA, Bes M, Brun Y, et al. Recommended minimal standards for description of new staphylococcal species. Int J Syst Evol Microbiol. Microbiology Society; 1999;49:489502.

[5] Carlton L. Gyles, John F. Prescott, J. Glenn Songer and COT. Pathogenesis of Bacterial Infections in Animals. 2010.

[6] Kloos WE, Schleifer KH. Genus IV. Staphylococcus Rosenbach 1884. Bergey's Man Syst Bacteriol. $1986 ; 2$.

[7] Nemati M, Hermans K, Lipinska U, Denis O, Deplano A, Struelens M, et al. Antimicrobial resistance of old and recent Staphylococcus aureus isolates from poultry: first detection of livestock-associated methicillin-resistant strain ST398. Antimicrob Agents Chemother. Am Soc Microbiol; 2008;52:3817-9.

[8] Van den Eede A, Martens A, Lipinska U, Struelens M, Deplano A, Denis O, et al. High occurrence of methicillin-resistant Staphylococcus aureus ST398 in equine nasal samples. Vet Microbiol. Elsevier; 2009;133:138-44.

[9] Kolawole DO, Shittu AO. Unusual recovery of animal staphylococci from septic wounds of hospital patients in Ile-Ife, Nigeria. Lett Appl Microbiol. Wiley Online Library; 1997;24:87-90.

[10] McNamee PT, Smyth JA. Bacterial chondronecrosis with osteomyelitis ('femoral head necrosis') of broiler chickens: a review. Avian Pathol. Taylor \& Francis; 2000;29:477-95.

[11] Devriese LA. A simplified system for biotyping Staphylococcus aureus strains isolated from different animal species. J Appl Microbiol. Wiley Online Library; 1984;56:215-20.

[12] De Neeling AJ, Van den Broek MJM, Spalburg EC, van Santen-Verheuvel MG, Dam-Deisz WDC, Boshuizen HC, et al. High prevalence of methicillin resistant Staphylococcus aureus in pigs. Vet Microbiol. Elsevier; 2007;122:366-72.

[13] Bannoehr J, Zakour NL Ben, Waller AS, Guardabassi L, Thoday KL, van den Broek AHM, et al. Population genetic structure of the Staphylococcus intermedius group: insights into agr diversification and the emergence of methicillin-resistant strains. J Bacteriol. Am Soc Microbiol; 2007;189:8685-92. 
[14] Hesselbarth J, Schwarz S. Comparative ribotyping of Staphylococcus intermedius from dogs, pigeons, horses and mink. Vet Microbiol. Elsevier; 1995;45:11-7.

[15] Mirt D. Lesions of so-called flank biting and necrotic ear syndrome in pigs. Vet Rec. 1999;144:92-6.

[16] Todar K. Todar's online textbook of bacteriology. University of Wisconsin-Madison Department of Bacteriology. 2005.

[17] Aires de Sousa M, Lencastre H. Bridges from hospitals to the laboratory: genetic portraits of methicillin-resistant Staphylococcus aureus clones. Pathog Dis. Wiley Online Library; 2004;40:101-11.

[18] Rooijakkers SHM, Van Kessel KPM, Van Strijp JAG. Staphylococcal innate immune evasion. Trends Microbiol. Elsevier; 2005;13:596-601.

[19] Urban CF, Lourido S, Zychlinsky A. How do microbes evade neutrophil killing? Cell Microbiol. Wiley Online Library; 2006;8:1687-96.

[20] Graves SF, Kobayashi SD, DeLeo FR. Community-associated methicillin-resistant Staphylococcus aureus immune evasion and virulence. J Mol Med. Springer; 2010;88:109-14.

[21] Zhu Y. Staphylococcus aureus virulence factors synthesis is controlled by central metabolism. The University of Nebraska-Lincoln; 2010.

[22] Novick RP. Autoinduction and signal transduction in the regulation of staphylococcal virulence. Mol Microbiol. Wiley Online Library; 2003;48:1429-49.

[23] Ma Y, Xu Y, Yestrepsky BD, Sorenson RJ, Chen M, Larsen SD, et al. Novel inhibitors of Staphylococcus aureus virulence gene expression and biofilm formation. PLoS One. Public Library of Science; 2012;7:e47255.

[24] Mirani ZA, Aziz M, Khan MN, Lal I, ul Hassan N, Khan SI. Biofilm formation and dispersal of Staphylococcus aureus under the influence of oxacillin. Microb Pathog. Elsevier; 2013;61:66-72.

[25] Olivier AC, Lemaire S, Van Bambeke F, Tulkens PM, Oldfield E. Role of rsbU and staphyloxanthin in phagocytosis and intracellular growth of Staphylococcus aureus in human macrophages and endothelial cells. J Infect Dis. The University of Chicago Press; 2009;200:1367-70.

[26] Yoon SH, Park Y-K, Lee S, Choi D, Oh TK, Hur C-G, et al. Towards pathogenomics: a web-based resource for pathogenicity islands. Nucleic Acids Res. Oxford University Press; 2006;35:D395-400.

[27] Yoon SH, Park Y-K, Kim JF. PAIDB v2. 0: exploration and analysis of pathogenicity and resistance islands. Nucleic Acids Res. Oxford University Press; 2014;43:D624-30.

[28] Yoon SH, Hur C-G, Kang H-Y, Kim YH, Oh TK, Kim JF. A computational approach for identifying pathogenicity islands in prokaryotic genomes. BMC Bioinformatics. BioMed Central; 2005;6:184.

Citation: Desiye Tesfaye Tegegne, "Virulence Factors and Pathogencity Islands in Staphylococcus Species: A Review" International Journal of Research Studies in Microbiology and Biotechnology (IJRSMB), vol. 6, no. 2, pp.14-20, 2020. Available: DOI: http://dx.doi.org/10.20431/2454-9428.0601002

Copyright: (c) 2020 Authors. This is an open-access article distributed under the terms of the Creative Commons Attribution License, which permits unrestricted use, distribution, and reproduction in any medium, provided the original author and source are credited. 